\title{
X-Ray Study of a Pentacyclic Partially Saturated Benzothiazolo[2,3-a]isoindolone
}

\author{
Kalevi Pihlaja, ${ }^{\star, a}$ Reijo Sillanpää, ${ }^{a}$ Géza Stájer ${ }^{b}$ and Samuel Frimpong-Manso ${ }^{b}$ \\ a Department of Chemistry, University of Turku, SF-20500 Turku, Finland and ${ }^{b}$ Institute of Pharmaceutical Chemistry, Albert \\ Szent-Györgyi Medical University, P.O.B. 121, H-6701 Szeged, Hungary
}

\begin{abstract}
Pihlaja, K., Sillanpää, R., Stájer, G. and Frimpong-Manso, S., 1992. X-Ray Study of a Pentacyclic Partially Saturated Benzothiazolo[2,3-a]isoindolone. - Acta Chem. Scand. 46: 1021-1023.
\end{abstract}

As part of our studies on various anorexigenic agents, the crystal structure of $12 \mathrm{~b}$ - $p$-tolyl-3,6-methano-2a,3,6,6a,7,$8,9,10,11,12,12 \mathrm{a}$-dodecahydro-3,1-benzoxazino[2,3-a]isoindol-8(12bH)-one was determined. ${ }^{1}$ The definite structures of such partly saturated condensed isoindolones are difficult to elucidate by means of NMR owing to severe signal overlap.

As a continuation of these studies ${ }^{1,2}$ we now report the crystal structure of 9,11b-diphenyl-8,11-methano$7 \mathrm{a}, 8,9,10,11,11 \mathrm{a}-\mathrm{h}$ exahydrobenzothiazolo[2,3-a] isoindol$7(11 \mathrm{bH})$-one (3) which was synthesized (Scheme 1) from 3-endo-benzoyl-6-exo-phenylbicyclo[2.2.1]heptane-2-endocarboxylic acid (1) and 2-aminothiophenol (2).

The presence of the saturated terminal bicycle can lead to various stereopositions of the two hetero-rings, i.e., the position of the 11b-phenyl group relative to the hydrogen atoms at the norbornane-pyrrolidine fusion can be different. Furthermore the location and steric arrangement of the phenyl group on the norbornane moiety needed to be proved.

\section{Experimental}

Crystal data for 3. $\mathrm{C}_{27} \mathrm{H}_{23} \mathrm{NOS}$, monoclinic, space group $P 2_{1} / a, a=13.949(4), b=10.691(3), c=15.203(3) \AA$, $\beta=110.00(2), V=2130.5(9) \AA^{3}, Z=4, D_{\mathrm{c}}=1.277 \mathrm{~g}$ $\mathrm{cm}^{-3}, \mu\left(\right.$ Mo $\left.K_{\alpha}\right)=1.62 \mathrm{~cm}^{-1}, T=296(1) \mathrm{K}$; colourless cubes.
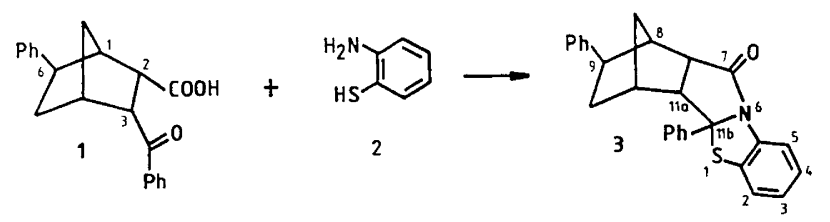

Scheme 1.

\footnotetext{
* To whom correspondence should be addressed.
}

Data collection, analysis and refinement. A Rigaku AFC5S diffractometer was used, with graphite-monochromated Mo $K_{\alpha}$ radiation $(\lambda=0.71069 \AA)$, in the $\omega-2 \theta$ scan mode, with an $\omega$ scan rate of $8.0^{\circ} \mathrm{min}^{-1}$ and a scan width of $(1.57+0.30 \tan \theta)$. The weak reflections $[I<10 \sigma(I)]$ were rescanned up to two times. The data obtained were corrected for Lorentz and polarization, but not for absorption effects. 3988 unique reflections were obtained $\left(2 \theta_{\max }=50^{\circ}\right)$. Direct methods and difference electron density $(\Delta \delta)$ calculations were used. Refinement of structural parameters was by full-matrix least-squares refinement, with non-hydrogen atoms anisotropic, and hydrogen atoms with fixed isotropic temperature parameters (1.2 times $B_{\text {eq }}$ of the carrying atom). In the final cycles, the 2261 data with $I>2 \sigma(I)$ yielded an $R$ value of $0.046\left(R_{\mathrm{w}}=0.051, w=\left[\sigma^{2}\left(F_{\mathrm{o}}\right)\right]^{-1}\right)$ for 340 parameters; maximum/minimum $\Delta \delta=0.19 /-0.25$ e $\AA^{-3}$.

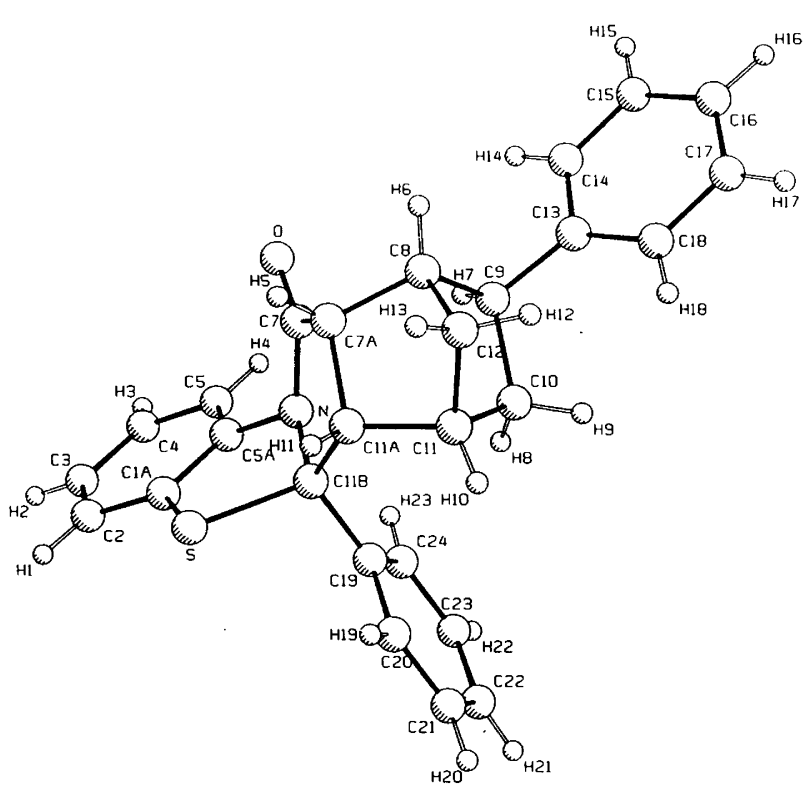

Fig. 1. Solid-state conformation of 3 by PLUTO drawing. 
Table 1. Positional parameters $\left(\times 10^{4}\right)$ and $B($ eq $)\left(\AA^{2}\right)$ for nonhydrogen atoms. ${ }^{a}$

\begin{tabular}{|c|c|c|c|c|}
\hline $\mathrm{S}$ & $1318.6(7)$ & $2593(1)$ & $744.3(6)$ & $3.87(4)$ \\
\hline 0 & $1123(2)$ & $6656(3)$ & $1144(2)$ & $4.9(1)$ \\
\hline $\mathbf{N}$ & $1666(2)$ & $4689(3)$ & $1709(2)$ & $3.0(1)$ \\
\hline C1A & $2365(3)$ & $3527(4)$ & $775(2)$ & $3.6(2)$ \\
\hline C2 & $3029(3)$ & $3333(5)$ & $282(3)$ & $4.6(2)$ \\
\hline C3 & $3789(4)$ & $4211(6)$ & $379(3)$ & $5.8(3)$ \\
\hline C4 & $3892(3)$ & $5247(5)$ & $933(3)$ & $5.3(2)$ \\
\hline C5 & $3219(3)$ & $5457(4)$ & $1424(3)$ & $4.1(2)$ \\
\hline C5A & $2462(3)$ & $4586(4)$ & $1327(2)$ & $3.4(2)$ \\
\hline C7 & $933(3)$ & $5625(4)$ & $1365(2)$ & $3.3(2)$ \\
\hline C7A & $-94(3)$ & $5117(4)$ & $1295(3)$ & $3.3(2)$ \\
\hline C8 & $-641(3)$ & $5733(4)$ & $1913(3)$ & $3.4(2)$ \\
\hline C9 & $152(3)$ & $5959(3)$ & $2905(2)$ & $3.2(1)$ \\
\hline C10 & 364(3) & $4609(4)$ & $3296(3)$ & $3.7(2)$ \\
\hline C11 & $-295(3)$ & $3779(4)$ & $2495(3)$ & $3.9(2)$ \\
\hline C11A & $87(3)$ & $3758(4)$ & $1654(2)$ & $3.2(2)$ \\
\hline C11B & $1216(2)$ & $3475(3)$ & $1779(2)$ & $2.9(1)$ \\
\hline C12 & $-1242(3)$ & $4612(4)$ & $2083(3)$ & $4.4(2)$ \\
\hline C13 & $-232(3)$ & $6899(3)$ & $3451(2)$ & $3.3(2)$ \\
\hline C14 & $-8(3)$ & $8151(4)$ & $3403(3)$ & $3.8(2)$ \\
\hline C15 & $-399(3)$ & $9064(4)$ & $3834(3)$ & $4.6(2)$ \\
\hline C16 & $-1017(4)$ & $8743(4)$ & $4324(3)$ & $5.0(2)$ \\
\hline C17 & $-1228(4)$ & $7505(5)$ & $4403(3)$ & $5.7(2)$ \\
\hline C18 & $-842(4)$ & $6591(4)$ & $3969(3)$ & $4.9(2)$ \\
\hline C19 & $1817(3)$ & $2748(3)$ & $2650(2)$ & $2.9(1)$ \\
\hline C2O & $1465(3)$ & $1585(4)$ & $2819(3)$ & $3.8(2)$ \\
\hline $\mathrm{C} 21$ & $1985(4)$ & $922(4)$ & $3614(3)$ & $4.4(2)$ \\
\hline $\mathrm{C} 22$ & $2868(3)$ & $1393(4)$ & $4255(3)$ & $4.3(2)$ \\
\hline $\mathrm{C} 23$ & $3229(3)$ & $2525(4)$ & $4089(3)$ & $4.3(2)$ \\
\hline C24 & $2709(3)$ & $3202(4)$ & $3292(3)$ & $3.5(2)$ \\
\hline
\end{tabular}

${ }^{a}$ E.s.d.s are given in parentheses.

Neutral atomic scattering and dispersion factors were taken from Ref. 3. All calculations were performed with TEXSAN-89 software, ${ }^{4}$ using a VAXSTATION 3520 computer. Figures were drawn with PLUTO $^{5}$ (cf. Fig. 1) software. The final atomic positional coordinates for nonhydrogen atoms are listed in Table 1, relevant bond lengths in Table 2 and relevant bond angles of non-hydrogen atoms in Table 3 and torsion angles in Table 4 . Lists of the final positional coordinates for hydrogen atoms, complete lists of bond lengths and angles and anisotropic thermal

Table 2. Relevant bond lengths $(\AA) .^{\text {a }}$

\begin{tabular}{llll}
\hline S-C1A & $1.756(4)$ & C7-C7A & $1.502(5)$ \\
S-C11B & $1.882(3)$ & C7A-C8 & $1.546(5)$ \\
O-C7 & $1.208(4)$ & C7A-C11A & $1.542(5)$ \\
N-C5A & $1.423(4)$ & C8-C9 & $1.554(5)$ \\
N-C7 & $1.397(4)$ & C8-C12 & $1.535(5)$ \\
N-C11B & $1.462(4)$ & C9-C10 & $1.550(5)$ \\
C1A-C2 & $1.392(5)$ & C9-C13 & $1.512(5)$ \\
C1A-C5A & $1-389(5)$ & C10-C11 & $1.533(5)$ \\
C2-C3 & $1.386(7)$ & C11-C11A & $1.544(5)$ \\
C3-C4 & $1.368(7)$ & C11-C12 & $1.539(5)$ \\
C4-C5 & $1.402(6)$ & C11A-C11B & $1.549(5)$ \\
C5-C5A & $1.378(5)$ & C11B-C19 & $1.517(4)$ \\
\hline
\end{tabular}

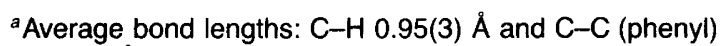
$1.379(5) \AA$.
Table 3. Relevant bond angles ( ${ }^{\circ}$ ) of non-hydrogen atoms.

\begin{tabular}{lrllr}
\hline C1A-S-C11B & $89.6(2)$ & C7A-C8-C9 & $108.7(3)$ \\
C5A-N-C7 & $118.4(3)$ & C7A-C8-C12 & $100.4(3)$ \\
C5A-N-C11B & $112.0(3)$ & C9-C8-C12 & $101.9(3)$ \\
C7-N-C11B & $112.7(3)$ & C8-C9-C10 & $101.9(3)$ \\
S-C1A-C2 & $127.0(3)$ & C8-C9-C13 & $111.6(3)$ \\
S-C1A-C5A & $112.8(3)$ & C10-C9-C13 & $117.7(3)$ \\
C2-C1A-C5A & $120.1(4)$ & C9-C10-C11 & $104.5(3)$ \\
C1A-C2-C3 & $117.9(4)$ & C10-C11-C11A & $112.6(3)$ \\
C2-C3-C4 & $122.0(4)$ & C10-C11-C12 & $100.7(3)$ \\
C3-C4-C5 & $120.5(5)$ & C11A-C11-C12 & $99.4(3)$ \\
C4-C5-C5A & $117.6(4)$ & C7A-C11A-C11 & $102.6(3)$ \\
N-C5A-C1A & $112.5(3)$ & C7A-C11A-C11B & $105.4(3)$ \\
N-C5A-C5 & $125.6(3)$ & C11-C11A-C11B & $121.6(3)$ \\
C1A-C5A-C5 & $121.9(3)$ & S-C11B-N & $103.1(2)$ \\
O-C7-N & $123.8(3)$ & S-C11B-C11A & $111.1(2)$ \\
O-C7-C7A & $127.2(4)$ & S-C11B-C19 & $107.2(2)$ \\
N-C7-C7A & $109.0(3)$ & N-C11B-C11A & $105.1(3)$ \\
C7-C7A-C8 & $117.4(3)$ & N-C11B-C19 & $113.3(3)$ \\
C7-C7A-C11A & $106.2(3)$ & C11A-C11B-C19 & $116.3(3)$ \\
C8-C7A-C11A & $104.0(3)$ & C8-C12-C11 & $94.5(3)$ \\
\hline
\end{tabular}

Table 4. Torsion angles ( ${ }^{\circ}$.

\begin{tabular}{|c|c|c|c|}
\hline$-C_{1} A-C 2-C 3$ & $177.7(3)$ & $\mathrm{C} 7-\mathrm{N}-\mathrm{C} 11 \mathrm{~B}-\mathrm{C} 11 \mathrm{~A}$ & $-12.8(3)$ \\
\hline$S-C 1 A-C 5 A-N$ & $-2.3(4)$ & $\mathrm{C} 7-\mathrm{N}-\mathrm{C} 11 \mathrm{~B}-\mathrm{C} 19$ & $-140.8(3)$ \\
\hline$S-C 1 A-C 5 A-C 5$ & $-178.3(3)$ & $\mathrm{C} 7-\mathrm{C} 7 \mathrm{~A}-\mathrm{C} 8-\mathrm{C} 9$ & $-42.7(4)$ \\
\hline$S-C 11 B-N-C 5 A$ & $-32.8(3)$ & C7-C7A-C8-C12 & $-149.2(3)$ \\
\hline $\mathrm{S}-\mathrm{C} 11 \mathrm{~B}-\mathrm{N}-\mathrm{C} 7$ & $103.7(3)$ & $C 7-C 7 A-C 11 A-C 11$ & 119.9(3) \\
\hline$S-C 11 B-C 11 A-C 7 A$ & $-98.4(3)$ & $C 7-C 7 A-C 11 A-C 11 B$ & $-8.3(3)$ \\
\hline$-C 11 B-C 11 A-C 11$ & $145.8(3)$ & $\mathrm{C} 7 \mathrm{~A}-\mathrm{C} 7-\mathrm{N}-\mathrm{C} 11 \mathrm{~B}$ & $7.6(4)$ \\
\hline $\mathrm{S}-\mathrm{C} 11 \mathrm{~B}-\mathrm{C} 19-\mathrm{C} 20$ & $-68.4(3)$ & C7A-C8-C9-C10 & $-71.2(4)$ \\
\hline$S-C 11 B-C 19-C 24$ & $111.9(3)$ & C7A-C8-C9-C13 & $162.4(3)$ \\
\hline $\mathrm{O}-\mathrm{C} 7-\mathrm{N}-\mathrm{C} 5 \mathrm{~A}$ & $-37.2(5)$ & $\mathrm{C} 7 \mathrm{~A}-\mathrm{C} 8-\mathrm{C} 12-\mathrm{C} 11$ & $56.1(3)$ \\
\hline $\mathrm{O}-\mathrm{C} 7-\mathrm{N}-\mathrm{C} 11 \mathrm{~B}$ & $-170.7(3)$ & $\mathrm{C} 7 \mathrm{~A}-\mathrm{C} 11 \mathrm{~A}-\mathrm{C} 11-\mathrm{C} 10$ & $-65.9(4)$ \\
\hline$-C 7-c$ & $-65.1(5)$ & $C 7 A-C$ & $39.8(3)$ \\
\hline $11 \mathrm{~A}$ & 179. & A-C11B-C19 & $138.6(3)$ \\
\hline $\mathrm{N}-\mathrm{C} 5 \mathrm{~A}-\mathrm{C}$ & & $\mathrm{C} 8-\mathrm{C} 7$ & $-4.6(4)$ \\
\hline $\mathrm{N}-\mathrm{C} 5 \mathrm{~A}-\mathrm{C} 5-\mathrm{C} 4$ & $-174.8(3)$ & $C 8-C 7 A-C 11 A-C 11 B$ & $-132.8(3)$ \\
\hline $\mathrm{N}-\mathrm{C} 7-\mathrm{C} 7 \mathrm{~A}-\mathrm{C} 8$ & $116.7(3)$ & C8-C9-C10-C11 & $1.4(4)$ \\
\hline $\mathrm{N}-\mathrm{C} 7-\mathrm{C} 7 \mathrm{~A}-\mathrm{C} 11 \mathrm{~A}$ & $1.0(4)$ & & \\
\hline$-S-C 1 A$ & $26.0(2)$ & $\mathrm{C} 8-$ & $85.4(5)$ \\
\hline $\mathrm{N}-\mathrm{C} 11 \mathrm{~B}-\mathrm{C} 11 \mathrm{~A}-\mathrm{C} 7 \mathrm{~A}$ & $12.4(3)$ & C8-C12-C11-C10 & $56.1(3)$ \\
\hline $\mathrm{N}-\mathrm{C} 11 \mathrm{~B}-\mathrm{C} 11 \mathrm{~A}-\mathrm{C} 11$ & $-103.3(4)$ & $\mathrm{C} 8-\mathrm{C}_{12}-\mathrm{C} 11-\mathrm{C} 11 \mathrm{~A}$ & $-59.2(3)$ \\
\hline $\mathrm{N}-\mathrm{C} 11 \mathrm{~B}-\mathrm{C} 19-\mathrm{C} 20$ & $178.6(3)$ & C9-C8-C7A-C11A & $74.2(4)$ \\
\hline $\mathrm{N}-\mathrm{C} 11 \mathrm{~B}-\mathrm{C} 19-\mathrm{C} 24$ & $-1.1(4)$ & $\mathrm{C} 9-\mathrm{C} 8-\mathrm{C} 12-\mathrm{C} 11$ & $-55.8(3)$ \\
\hline $1 \mathrm{~A}$ & 138. & & $68.6(4)$ \\
\hline $1 \mathrm{~A}-\mathrm{S}-\mathrm{C} 11 \mathrm{~B}-\mathrm{C} 19$ & $-93.8(2)$ & $\mathrm{C} 9-\mathrm{C} 1$ & $-36.3(4)$ \\
\hline $\mathrm{C} 1 \mathrm{~A}-\mathrm{C} 2-\mathrm{C} 3-\mathrm{C} 4$ & $-0.5(7)$ & $\mathrm{C} 9-\mathrm{C} 13$ & $175.3(3)$ \\
\hline $\mathrm{C} 1 \mathrm{~A}-\mathrm{C} 5 \mathrm{~A}-\mathrm{N}-\mathrm{C} 7$ & $-109.2(4)$ & $\mathrm{C} 9-\mathrm{C} 13$ & $-175.5(4)$ \\
\hline $\mathrm{C} 1 \mathrm{~A}-\mathrm{C} 5 \mathrm{~A}-\mathrm{N}-\mathrm{C} 11 \mathrm{~B}$ & $24.6(4)$ & $\mathrm{C} 10-\mathrm{C} 9-\mathrm{C} 8-\mathrm{C} 12$ & 34.3(3) \\
\hline $\mathrm{C} 1 \mathrm{~A}-\mathrm{C} 5 \mathrm{~A}-\mathrm{C} 5-\mathrm{C} 4$ & $0.7(6)$ & $\mathrm{C}_{10}-\mathrm{C9}_{-} \mathrm{C}_{13}-\mathrm{C}_{14}$ & $151.4(4)$ \\
\hline$C 2-C 1 A-S-C 11 B$ & $168.9(3)$ & C10-C9-C13-C18 & $-31.8(6)$ \\
\hline$-\mathrm{C} 5 \mathrm{~A}-\mathrm{C} 5$ & $-1.5(5)$ & $\mathrm{C} 10-$ & $51.2(5)$ \\
\hline C2-C3- & $-0.2(7)$ & C11-C10-C9-C13 & $123.7(3)$ \\
\hline C3-C2-C1A-C5A & $1.4(6)$ & $\mathrm{C} 11-\mathrm{C} 11 \mathrm{~A}-\mathrm{C} 11 \mathrm{~B}-\mathrm{C} 19$ & $22.9(5)$ \\
\hline C3-C4-C5-C5A & $0.2(6)$ & $\mathrm{C} 11 \mathrm{~A}-\mathrm{C} 7 \mathrm{~A}-\mathrm{C} 8-\mathrm{C} 12$ & $-32.3(4)$ \\
\hline $\mathrm{C} 5-\mathrm{C} 5 \mathrm{~A}-\mathrm{N}-\mathrm{C} 7$ & $66.6(5)$ & $\mathrm{C} 11 \mathrm{~A}-\mathrm{C} 11 \mathrm{~B}-\mathrm{C} 19-\mathrm{C} 20$ & $56.6(4)$ \\
\hline $\mathrm{C} 5-\mathrm{C} 5 \mathrm{~A}-\mathrm{N}-\mathrm{C} 11 \mathrm{~B}$ & $-159.6(3)$ & $\mathrm{C} 11 \mathrm{~A}-\mathrm{C} 11 \mathrm{~B}-\mathrm{C} 19-\mathrm{C} 24$ & $-123.1(4)$ \\
\hline $\mathrm{C} 5 \mathrm{~A}-\mathrm{N}-\mathrm{C} 7-\mathrm{C} 7 \mathrm{~A}$ & $141.1(3)$ & $\mathrm{C} 11 \mathrm{~B}-\mathrm{C} 11 \mathrm{~A}-\mathrm{C} 11-\mathrm{C} 12$ & $157.0(3)$ \\
\hline $\mathrm{C} 5 \mathrm{~A}-\mathrm{N}-\mathrm{C} 11 \mathrm{~B}-\mathrm{C} 11 \mathrm{~A}$ & $-149.3(3)$ & $\mathrm{C} 11 \mathrm{~B}-\mathrm{C} 19-\mathrm{C} 20-\mathrm{C} 21$ & $-178.6(3)$ \\
\hline & & $\mathrm{C} 11 \mathrm{~B}-\mathrm{C} 19-\mathrm{C} 24-\mathrm{C} 23$ & $178.7(3)$ \\
\hline C5A-C1A-S-C11B & $-14.5(3)$ & $\mathrm{C} 12-\mathrm{C} 8-\mathrm{C} 9-\mathrm{C} 13$ & $-92.1(3)$ \\
\hline
\end{tabular}


parameters and observed and calculated structural factors are available from the authors on request.

\section{Results and discussion}

The X-ray analysis indicates that the phenyl substituent on the norbornane ring occupies the 9-exo-position. It was important to establish this because the phenyl group in the initial reactant 1, prepared from bicyclo[2.2.1] hept-5-ene2,3-dicarboxylic acid anhydride by addition and acylation of benzene in one step, could, in principle, be located at C10. The exo position of this phenyl group is not surprising since the saturation of the norbornene double bond generally results in exo substitution.

The endo phenyl group at $\mathrm{C} 11 \mathrm{~B}$ and the hydrogen atoms at $\mathrm{C} 7 \mathrm{~A}$ and $\mathrm{C} 11 \mathrm{~A}$, namely $\mathrm{H} 7 \mathrm{~A}$ and $\mathrm{H} 11 \mathrm{~A}$, are trans relative to the pyrrolidine ring. Fig. 1 shows the perspective view of the molecule. ${ }^{5}$ One of the heterocyclic fivemembered rings $(\mathrm{C} 11 \mathrm{~B}, \mathrm{~S}, \ldots, \mathrm{N})$ attains an envelope form $\left(E_{1}\right)$ with the puckering parameters $Q=0.327(1) \AA$ and $\varphi=179.5(2)^{\circ}$ and the ring torsion angles starting from
C5A-N-C11B-S of $-38.8(3), 26.0(2),-14.5(3),-2.3(4)$ and $24.6(4)^{\circ}$, respectively. The conformation of the second five-membered heterocycle $(\mathrm{C} 11 \mathrm{~B}, \mathrm{~N}, \ldots, \mathrm{C} 11 \mathrm{~A})$ is a flattened ${ }^{1} E$ envelope with the puckering parameters $Q=0.125(2) \AA$ and $\varphi=353.9(7)^{\circ}$ and the ring torsion angles starting from $\mathrm{C} 11 \mathrm{~A}-\mathrm{C} 11 \mathrm{~B}-\mathrm{N}-\mathrm{C} 7$ of $-12.8(3)$, $7.6(4), 1.0(4),-8.3(3)$ and $12.4(3)^{\circ}$, respectively.

\section{References}

1. Stájer, G., Sillanpää, R. and Pihlaja, K. To be submitted.

2. Kálmán, A., Argay, Gy., Stájer, G. and Bernáth, G. J. Mol. Struct. 248 (1991) 167 and references therein.

3. International Tables for X-Ray Crystallography, Kynoch Press, Birmingham, England 1974, Vol. IV.

4. TEXSAN: Single Crystal Structure Analysis Software, Version 5.0, Molecular Structure Corporation, The Woodlands, Texas 1989.

5. Motherwell, W. D. S. and Clegg, W. PLUTO 78. A Program for Plotting Crystal and Molecular Structures, University of Cambridge, England 1978.

Received March 4, 1992. 